\title{
Les centres urbains en Algérie : comment concilier l'attractivité et la mobilité à travers la gestion du transport urbain ? Cas de la ville de Bejaia
}

\author{
Urban centres in Algeria: \\ how to reconcile attractivity and mobility through the management of urban transport? \\ Case of the city of Bejaia
}

\author{
Slimane Merzoug \\ (C) IFSTTAR et Éditions NecPlus 2016
}

\begin{abstract}
Résumé La carence en matière d'aménités urbaines dans les zones périphériques aux grands centres urbains en Algérie et la concentration des emplois et des fonctions administratives dans ces derniers engendre une forte demande de déplacement qui dépasse les capacités de l'offre. Afin d'améliorer cette offre, l'État a marqué son retour à travers la création des entreprises publiques de transport urbain par bus et le développement du transport de masse tel que le métro et le tramway. À l'instar des autres grandes villes, Bejaia devrait être doté d'un tramway à l'horizon 2020, outre un plan de circulation qui prévoit divers aménagements.

L'objet de cet article est d'analyser la capacité de la nouvelle offre de transport urbain à améliorer les déplacements quotidiens dans cette ville. A cet effet, une étude statistique est réalisée sur les données des enquêtes Tous Modes Motorisés (TMM) et Origine/Destination (2013) et l'Enquête Ménage Déplacement (2006), en tenant compte de l'offre actuelle et nouvelle de transport urbain. Ainsi, cette étude conclut que les projets urbains prévus sont susceptibles de produire des impacts positifs en matière de desserte de la demande et d'atténuation des effets environnementaux, mais la participation citoyenne, l'intermodalité et le partage modal des espaces de circulation et de stationnement sont négligés ou sous-estimés ce qui pourrait empêcher l'optimisation du système de transport dans cette ville.
\end{abstract}

Slimane Merzoug $(\bowtie)$

Université de Bejaia, faculté des SECG, Laboratoire d'économie et développement 06000 Bejaia, Algérie

e-mail: merzougslimane@yahoo.fr
Mots-clés nouvelle offre de transport - centre urbain . déplacements quotidiens · transport urbain - ville de Bejaia

Abstract The urban amenities deficiency in zones outlying the big cities in Algeria as well as job and administrative office concentration there, generates a high transportation demand which exceeds supply capacities. In order to improve this offer, the State, consequently to a period of liberalization since 1980s, marked its comeback through the creation of public companies specialized in urban transportation by bus and the developing of the mass transportation like subway and tramway systems. Like other big towns, Bejaia should be equipped with a tramway on the horizon of 2020 in addition to a traffic planning which considers many rearrangements.

The purpose of this paper is to examine the new urban transportation offer ability to improve the daily mass traffic within this town. To that end, a statistical study based on data from the "All Motorized Modes and Origin/destination" (2013) and the "households mobility" (2006) surveys is carried out, taking into account both the current and the new offer on urban transportation. The study concludes that the urban projects planned for are likely to bring about favourable effects as regards serving the demand and softening environmental impacts. However, citizen participation, inter-modality and traffic and parking spaces modal-sharing are neglected or underrated, so that this could prevent the optimization of the transportation system in this town.

Keywords Transportation new offer - Urban centre - Daily mobility - Urban transportation - Town of Bejaia 


\section{Introduction}

L'urbanisation ne cesse de prendre de l'ampleur en Algérie avec une population urbaine qui constitue deux tiers de la population totale du pays ${ }^{1}$. Le milieu urbain et la population urbaine sont définis en Algérie sur la base d'une stratification légale et statistique (Tableau1).

À l'instar de toutes les villes, la ville de Bejaia connaît une forte densité et un étalement urbain dus à une croissance naturelle et à un exode rural; à la mobilité des résidents s'ajoute une mobilité quotidienne créée par les flux de population en provenance des communes limitrophes. La carence en matière d'aménités urbaines dans ces dernières et la concentration des emplois et des fonctions administratives dans cette ville expliquent sa forte attractivité.

En plus de la population résidente qui est de 184000 habitants $^{2}$ et de près de 40000 étudiants répartie à travers les différentes résidences universitaires, la ville accueille quotidiennement des dizaines de milliers de personnes qui se déplacent pour divers motifs : travail, études, démarches administratives, achats, loisirs... etc. En conséquence, cette attractivité altère la qualité d'accessibilité et réduit l'efficacité des déplacements au centre urbain. La faible efficacité s'explique par les faibles fréquences de service, l'augmentation des temps d'attentes aux arrêts et du coût du temps de déplacement. L'offre actuelle de transport collectif est mise en cause pour son incapacité à répondre à la forte croissance de la demande de déplacements qui est amplifiée par l'étalement urbain. Cet étalement mal maitrisé qui fait suite à une forte pression démographique a concouru à limiter le service public du transport collectif. Un tel effet négatif sur le transport urbain est une caractéristique de pays en développement [1], alors que la périurbanisation dans les pays développés a renforcé la dépendance à l'automobile $[2 ; 3]$. Sur le pourtour méditerranéen, Godard parle de crise de transport collectif dans toutes les villes du sud, ce qui a favorisé l'émergence de transport artisanal [4].

De plus, comme toutes les villes algériennes, la ville de Bejaia s'est développée autour d'une unique fonction : habiter ; tandis que les autres fonctions, notamment la circulation ont été négligées [5]. Afin de rattraper le retard et d'aller vers une mobilité durable, deux projets sont envisagés ; il s'agit d'un plan de circulation qui ambitionne de favoriser les modes de transport collectif et les modes actifs, et d'une nouvelle offre de transport de masse constituée par le projet de tramway. Le choix

\footnotetext{
${ }^{1}$ La part de la population urbaine est de $14 \%$ en 1886 , de $31 \%$ en 1966 et de $66 \%$ en 2008 (ONS, 2011).

${ }^{2}$ Selon une estimation de la direction de la planification et de l'aménagement du territoire de la wilaya de Bejaia, 2014. Toutefois, le dernier recensement général de la population et de l'habitat (RGPH de 2008) a donné un chiffre de 176139 habitants.
}

de ces types de projets est motivé par leurs capacités à répondre à la forte demande de déplacement et par leurs rôles dans la structuration et le fonctionnement de la ville $[6 ; 7]$. Toutefois, plusieurs études empiriques sur l'impact des transports de masse (BHLS ${ }^{3}$ et tramway) soulignent le comportement inerte des citoyens à l'utilisation de la voiture particulière et le faible effet de ces modes de transport sur la mobilité durable $[8 ; 9 ; 10]$. D'une façon globale, la ville est dédiée à l'automobile après la destruction de la ville pédestre, ce qui détermine fortement la structure et le choix des déplacements [11].

Les projets prévus dans la ville de Bejaia ont été précédés par la création d'une entreprise publique de transport urbain $\left(\right.$ ÉTUB $\left.^{4}\right)$ par bus en 2010. On assiste alors à un retour massif de l'État après une période de forte libéralisation qui a été motivée par l'atteinte d'un objectif social: la résorption du chômage qui a touché une large couche de la population après la crise des années 1980 [4; 5]. La libéralisation qui a ouvert la voie aux opérateurs artisanaux, ainsi que les différents plans d'aménagement local mis en place depuis l'indépendance n'ont pas répondu aux attentes des usagers des transports et ont comme effet le déclin de l'usage du transport collectif au profit de l'automobile. Il y a lieu de noter que la forte diffusion de l'automobile ${ }^{5}$ constitue un concurrent au transport collectif qui est délaissé par les usagers, en raison de son faible niveau de service et de l'absence d'une politique de gestion de l'espace urbain.

La nouvelle offre de transport n'est pas accompagnée d'une réorganisation des transports traditionnels qui sont mieux adaptés aux quartiers périphériques englués par une urbanisation informelle. Plus précisément, l'intermodalité qui se définit par l'organisation des correspondances et l'intégration tarifaire n'est pas prise en compte dans les nouveaux projets pour améliorer la fréquentation des transports collectifs. En clair, faute d'études préalables les deux projets n'ont que peu pris en compte la structure des déplacements et le transport collectif actuel. Ainsi, ces deux projets en cours qui annoncent le fort retour de l'État sont-ils en mesure d'améliorer les déplacements quotidiens dans cette ville ? Autrement dit, ces projets qui

\footnotetext{
${ }^{3}$ Bus with a High Level of Service: Bus avec un haut niveau de service. ${ }^{4}$ Établissement de transport urbain de Bejaia.

${ }^{5}$ Les statistiques de la gendarmerie nationale indiquent que le parc national d'automobile a connu une augmentation de $62 \%$ pendant ces dix dernières années (2004/2013), il est passé de 4482090 voitures en 2004 à 7286541 en 2013. La même source montre que la voiture particulière est celle qui a connu le plus grand taux de croissance. À titre indicatif, rien qu'au niveau de la wilaya de Bejaia il y a eu l'acquisition de 78902 véhicules de tourisme pendant la période de 2004 à 2013 sur un total de 124138 automobiles tous modes confondus (direction des transports de la wilaya, 2014).
} 
Tableau 1 Stratification des agglomérations urbaines en Algérie

Deux types de stratification des agglomérations urbaines sont adoptés en Algérie. Il s'agit des stratifications de type légal et statistique : 1. Les strates légales

La stratification légale est définie par les lois suivantes : $N^{\circ}$ 2001-20 du 12 décembre 2001 relative à l'aménagement et au développement durable du territoire ; $\mathrm{N}^{\circ}$ 2006-06 du 20 février 2006 portant loi d'orientation de la ville. Les dispositions de ces lois sont résumées dans le tableau suivant :

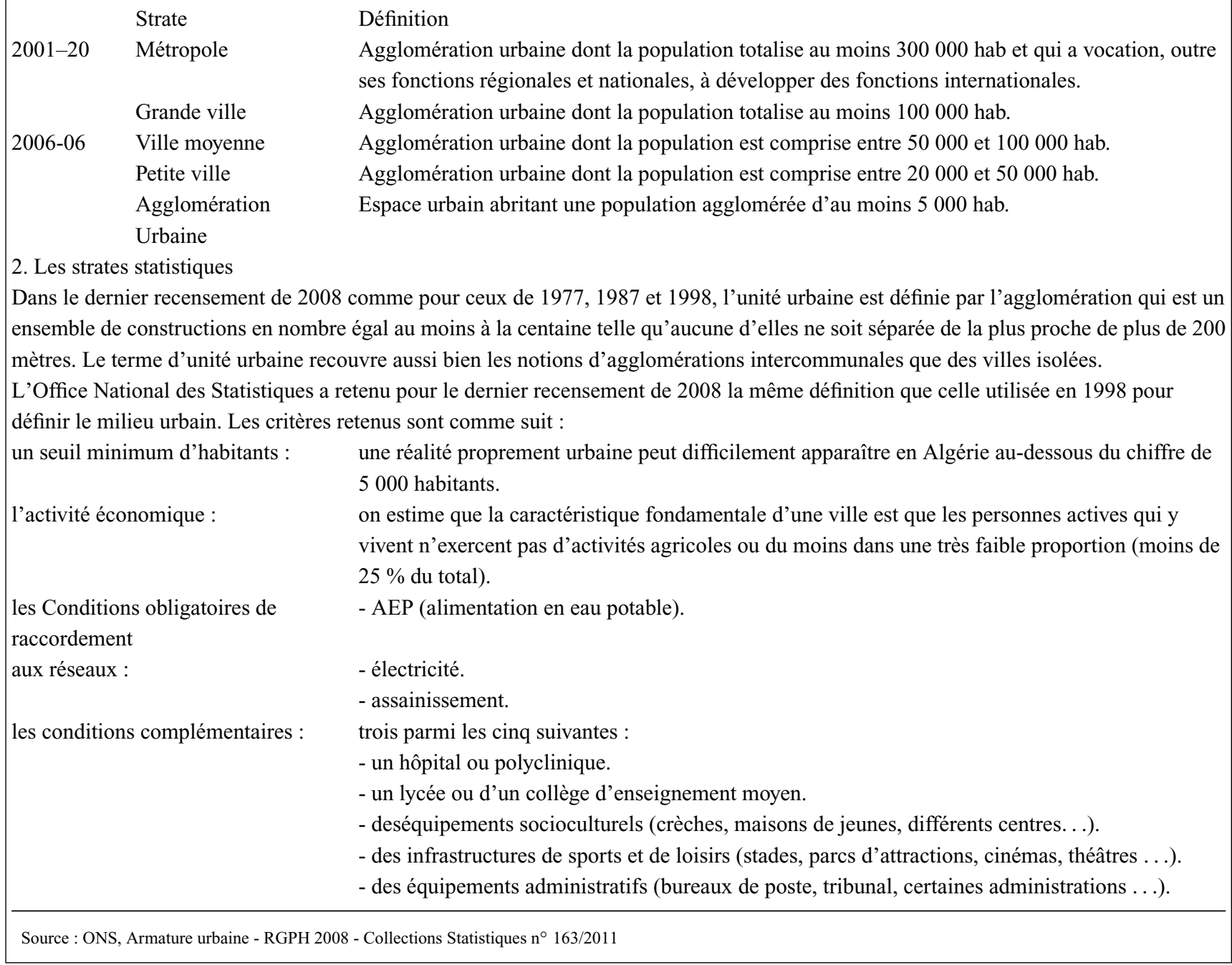

visent l'amélioration des déplacements urbains ont-ils pris en compte la structure modale actuelle et les motifs de déplacement au sein de la ville de Bejaia?

À cet effet, notre démarche est basée sur l'analyse de la structure de la demande de déplacement dans le centre urbain. Il s'agit, en premier lieu, de déterminer la structure des déplacements par modes sur les principales artères de l'agglomération et les flux d'entrée et de sortie de la ville, ainsi que les motifs de déplacement. L'objectif est de souligner quelle est la part de la voiture particulière et quels sont les motifs de déplacement qui sont susceptibles d'être orientés vers le transport collectif. Les données statistiques exploitées à cet effet sont tirées des enquêtes effectuées en 2013 par le Bureau d'études des transports (BETUR), filiale de l'Entreprise Métro d'Alger (EMA) et de l'enquête ménage déplacement (2006). En second lieu, nous avons analysé la capacité des transports traditionnels et du nouveau mode à répondre aux objectifs d'accessibilité et de mobilité dans un contexte de forte utilisation de la voiture particulière. Par ailleurs, en référence à quelques études sur l'impact des tramways en exploitation dans d'autres villes algériennes, nous avons tenté d'identifier quelques éléments qui doivent être pris en compte pour l'optimisation du système de transport dans cette ville. 
La présentation de nos analyses est faite à travers trois parties. La première partie explique les facteurs d'attractivité de la ville de Bejaia, donne une synthèse des dysfonctionnements actuels du système de transport urbain et les problèmes engendrés par la mobilité quotidienne des résidents comme des non-résidents et enfin, présente les solutions envisagées par les autorités (tramway et plan de circulation). La seconde partie est réservée à une analyse statistique de la structure de la demande, plus particulièrement sur les motifs et les modes de déplacement. Enfin, la dernière partie traite la capacité de la nouvelle offre de transport à répondre aux attentes de déplacements.

\section{Centre urbain de Bejaia : entre attractivité et problèmes de mobilité}

Le centre urbain et la ville de Bejaia désignent le chef-lieu de la commune de Bejaia. L'attractivité de cette ville est appréciée dans la présente étude par des dizaines de milliers de personnes qui sont attirées par les diverses fonctions qu'offre la ville. Les problèmes de mobilités engendrés par cette attractivité ont fait réagir les autorités par des actions qui visent à améliorer le système de transport.

\subsection{Ville de Bejaia : espace attractif}

La ville de Bejaia est le chef-lieu de la commune et de la wilaya. Elle est située au nord de l'Algérie, quelque 250 kilomètres à l'est de la capitale. C'est une ville méditerranéenne très ancienne qui est aujourd'hui parmi les villes les plus dynamiques, avec une activité industrielle et commerciale très importante. La présence d'un port qui est classé deuxième en Algérie en termes de tonnage constitue un facteur important de fixation des activités économiques et de la population dans cette ville. Sa population dépasse 184 000 habitants $^{6}$ et accueille quotidiennement des dizaines de milliers de personnes en provenance des communes limitrophes à cause de son statut de chef-lieu ${ }^{7}$ de wilaya qui concentre les plus importants services administratifs et commerciaux et une grande partie des emplois. Par l'effet d'une croissance naturelle et d'un exode rural massif, la population de la ville de Bejaia a augmenté de $56 \%$ de 1987 à 2013 (Figure 1). À cette concentration de la population dont la mobilité est amplifiée par l'étalement urbain, s'ajoutent des flux en provenance des communes limitrophes qui sont répartis entre les trois entrées de la ville constituées par les routes nationales 9, 12 et 24 .

\footnotetext{
${ }^{6}$ Nombre d'habitants de la commune de Bejaia au 31 décembre 2013. ${ }^{7} \mathrm{La}$ ville comprend des monuments historiques précoloniaux et des quartiers occidentaux de l'époque coloniale à qui s'ajoutent des extensions réalisées après l'indépendance.
}

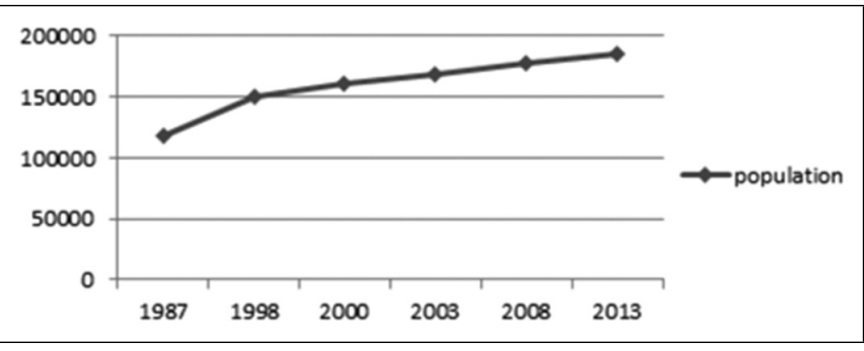

Graphe 1. Évolution de la population de la ville de Bejaia, 1987/2013

Source : Annuaire statistique de la wilaya de Bejaia, diverses années.

Bejaia est aussi une ville qui dispose d'une université constituée de filières très diverses et accueille 41698 étudiants ${ }^{8}$. Les déplacements de ces derniers entre une dizaine de résidences éparpillées à travers la ville vers les deux pôles universitaires d'enseignement se font par un transport spécialisé pris en charge par les œuvres universitaires. D'autres types de transports spécialisés dans le ramassage des employés des grandes entreprises publiques et privées (Sonatrach, Sonelgaz, Cevital, ENTP, ...) sont aussi présents dans cette ville. Ces transports spécialisés qui ne sont pas traités dans cette étude sont ancrés en Algérie depuis de nombreuses années.

Ses atouts en termes de dynamique économique et universitaire font de la région de Bejaia un lieu propice pour l'émergence d'une nouvelle génération d'entrepreneurs assez importante ${ }^{9}$. La forte présence du secteur privé qui couvre une majorité des besoins socio-économiques de la région témoigne de cette émergence des entrepreneurs dans les activités de production, mais aussi de services. C'est surtout dans les transports collectifs urbains et interurbains (dessertes entre les communes limitrophes et le centre urbain de Bejaia) que la présence des opérateurs privés est plus notable. Ces privés constituent une multitude de microentreprises qui assurent la quasi-totalité des déplacements urbains et interurbains depuis 1987, date de la libéralisation $\mathrm{du}$ secteur et du désengagement des entreprises publiques qui étaient en déficit chronique (entreprise communale de transport de Bejaia et entreprise publique de transport de voyageurs). La création des entreprises privées de transport a été encouragée par les autorités via l'octroi de crédits bancaires bonifiés et des exonérations fiscales à destination des jeunes chômeurs. Le transport public urbain est donc considéré par les autorités comme un moyen de lutte

\footnotetext{
${ }^{8}$ Selon l'annuaire statistique de la wilaya de Bejaia, 2014.

${ }^{9}$ La région de Bejaia concentre le plus important pôle industriel de l'Algérie dans le domaine de l'agroalimentaire. Des multinationales dans ce domaine industriel sont installées dans cette région et approvisionnent une grande partie du marché national et même contribuent à la diversification des exportations hors hydrocarbures.
} 
contre le chômage qui était très élevé à cette période, en raison de la poussée démographique des années 1970 et des réformes du secteur public suite à l'effondrement du prix du pétrole en 1986. Au grand bonheur des habitants de la ville, la libéralisation a permis de répondre aux besoins de déplacements après une période de très faible couverture de l'espace urbain par les transporteurs publics; elle a aussi favorisé la création d'emplois directs constitués au moins par deux chauffeurs et deux receveurs pour un simple minibus. Toutefois, les problèmes de mobilité sont vite apparus avec la forte croissance de la demande qui dépasse l'offre, mais aussi par un fort recours à la voiture particulière. Encouragés par les augmentations des salaires avec des effets rétroactifs sur au moins deux années ${ }^{10}$, les ménages algériens se sont rués vers la découverte $\mathrm{du}$ confort, d'autonomie et de souplesse que permet la voiture. Par ailleurs, plusieurs facteurs socio-économiques (pouvoir d'achat, prix du carburant, flexibilité, confort...) et d'aménagement de l'espace urbain qui ne favorisent pas le transport collectif et les modes actifs (marche à pied et vélo) expliquent l'engouement et le recours à l'utilisation de la voiture particulière.

Ces problèmes de mobilité qui feront l'objet de la partie suivante ne peuvent être corrigés que par une politique de transport durable ${ }^{11}$.

\subsection{Caractéristiques du transport urbain et problèmes de mobilité : congestion, stationnement, bruit et pollution}

Depuis sa libéralisation en 1987, le transport urbain est assuré dans sa quasi-totalité par les opérateurs privés. Ces derniers regroupent des entreprises unipersonnelles aux moyens très limités et fournissant une faible qualité de service. Le type de transport qu'ils offrent nous l'avons qualifié de traditionnel, en raison de la structure très limitée de leur équipement (Autocars de 50 places, Minibus de 25-35 places et Fourgons de 11-22 places) et d'une gestion aléatoire qui ne respecte pas les horaires, le confort et la sécurité des passagers, mais aussi des piétons. Tous ces dysfonctionnements sont le résultat d'une ouverture du marché au privé sans la mise en place d'un cadre de régulation qui définit les règles minimales d'exploitation et de concurrence.

\footnotetext{
${ }^{10}$ Les années 2010 et 2011 sont marquées par une augmentation conséquente des salaires, notamment de la fonction publique (éducation, enseignement supérieur, santé, administrations, services de sécurité et de police) avec des effets parfois rétroactifs à partir de 2008 .

${ }^{11}$ Les politiques de transport durable sont celles qui visent à promouvoir les transports les moins polluants, les moins encombrants, les moins consommateurs d'énergie, et ayant moins d'impact sur le paysage naturel et artificiel... etc. Ces vertus sont particulièrement présentes dans les modes « doux » et les transports collectifs.
}

Afin d'améliorer la mobilité urbaine et d'inciter les opérateurs privés à améliorer leur offre, les autorités ont mis en place, depuis quelques années, une nouvelle offre de transport constituée par des autobus de 100 places qui sont exploités par l'ÉTUB. L'opération s'inscrit dans le cadre du programme national qui vise à réhabiliter le secteur public avec un retour de l'État dans l'exploitation du transport urbain à l'échelle nationale [12].

À l'heure actuelle, les opérateurs privés représentent une part très importante du marché des transports urbains. Sur un total de 16282 places offertes par l'ensemble des opérateurs, le privé fournit 13482 places soit $82,8 \%$ et les $17,2 \%$ restants sont assurés par l'ÉTUB. À l'exception de la capitale, Alger, dans laquelle l'opérateur public est dominant, dans toutes les autres villes algériennes le transport en commun est dans sa quasi-totalité assuré par les opérateurs privés [13].

En termes de structure des investissements, les opérateurs privés possèdent un nombre de véhicules très limité, soit un opérateur pour 1,06 unité. La capacité moyenne de ces derniers est de 39,77 places avec une prépondérance des minicars de 35 places. En grande partie, ces opérateurs assurent le service de transport entre les quartiers périphériques et le centre-ville et opèrent sur un trajet moyen de 5,27 km. Quant à l'ÉTUB qui relie la nouvelle gare routière située à proximité des points d'accès de la ville (RN 9 et 12) aux principaux quartiers, il dessert surtout la plaine (couronne interne) puisque sa flotte d'autobus de grande taille ne peut pas accéder aux quartiers situés sur les hauteurs via des rues en majorité exiguës et souvent impraticables. La flotte de cette compagnie publique est composée de 28 autobus qui effectuent un trajet moyen de $9,94 \mathrm{~km}$ et offrent 2800 places.

Au-delà des caractéristiques des lignes et des opérateurs qui assurent le service de transport en commun dans la ville de Bejaia, l'organisation et la gestion de celui-ci sont en déphasage par rapport à la croissance de la demande de transport provoquée par les mutations démographiques et urbanistiques. Diverses lacunes du système de transport en matière d'organisation et de gestion peuvent être résumées dans les éléments suivants :

- fixation des prix du service à $15 \mathrm{DA}^{12}$ payables uniquement en espèce et ce quelque soit le trajet. Par ailleurs, il n'existe aucune politique de fidélisation et de facilitation pour les plus démunis ou les écoliers sous forme de cartes d'abonnement ou de tarifs réduits ;

- les arrêts de bus sont localisés la où il est possible, pas aux lieux nécessaires ${ }^{13}$, et dépourvus d'indications telles que

\footnotetext{
${ }^{12}$ Dinar algérien : unité monétaire algérienne. Selon le taux de change en cours au mois d'avril 2015, la valeur de 15 DA avoisine les $0,13 €$. ${ }^{13} \mathrm{~L}$ 'urbanisation non contrôlée dans cette ville laisse peu de possibilités pour une localisation rationnelle et pour étudier des arrêts de bus en fonction de la demande de transport.
} 
les horaires, les destinations et les numéros de lignes. Ces arrêts sont dans certains cas privés de plaques signalant leur existence, ainsi que d'abri bus pour les usagers ;

- une urbanisation anarchique qui affecte la qualité des routes, qui sont en plusieurs endroits impraticables, notamment dans les quartiers périphériques. Cette situation affecte la qualité des déplacements motorisés et ne facilite pas la mise en place d'une offre de transport de proximité, ce qui se traduit par le rallongement des distances parcourues à pied pour rejoindre les arrêts de bus ;

- le non-respect de la réglementation par les opérateurs en matière d'horaires de travail, de régularité de service. Sans formation spécialisée, ces opérateurs manquent de professionnalisme et adoptent des règles de conduite contraires à la réglementation telle que par exemple le changement d'itinéraire ou des demi-tours effectués au milieu du trajet pour assurer un remplissage maximum de leur bus.

En somme, cette inefficacité d'offre de transport collectif dans la ville de Bejaia rend les déplacements urbains aléatoires avec des délais longs et coûteux, notamment pour les plus démunis et les écoliers. De leur côté, les modes doux ne sont pas facilités en raison d'insuffisance d'espaces réservés. La marche à pied qui représente la moitié des déplacements en milieu urbain selon l'EMD de 2006 n'est donc pas favorisée par des plans d'aménagement. Elle représente toutefois une grande partie de la solution des problèmes de mobilité dans la ville de Bejaia, avec une superficie limitée à $120,2 \mathrm{~km}^{2}$ et une forte concentration urbaine.

Faute d'une gestion urbaine des espaces qui veille à un meilleur partage entre les différents modes de déplacement, la ville est livrée à la voiture particulière qui l'asphyxie. De son côté, le transport en commun est englué (voir Figure 2) dans des embouteillages interminables qui affectent son exploitation commerciale et sa convenance comme mode de déplacement privilégié. À l'heure actuelle, l'offre de transport collectif traditionnel (petit bus, fourgon, taxi) ne répond pas aux objectifs d'accessibilité en termes de qualité (mauvaise articulation dans le réseau urbain, absence d'intégration tarifaire, temps de déplacement long et aléatoire) et en quantité de service (surcharge des véhicules, notamment en heure de pointe, licences d'exploitation accordées sans la prise en compte des besoins de déplacement par ligne). Certes, la libéralisation du secteur en 1987 a permis la création de centaines ${ }^{14}$ de micro-entreprises privées, mais cette

\footnotetext{
${ }^{14}$ Le nombre d'opérateurs privés est de 320 pour 339 véhicules (minibus et fourgons) en 2014, selon la direction des transports de la wilaya de Bejaia (autorité locale chargée du transport urbain).
}

ouverture n'est pas encadrée ce qui a donné lieu à un service de transport urbain moins efficace. Dite autrement, la libéralisation a permis de répondre à un besoin ponctuel de transport, mais elle a créé en parallèle divers problèmes de mobilité (congestion, accident de la circulation, bruit et dégradation des conditions de vie, pollution et consommation d'énergie) qui engendrent un coût économique et social important pour toute la communauté locale et nationale.

La forte diffusion de l'automobile a laminé la progression des transports collectifs et a provoqué la désaffection des modes actifs [14]. En plus de la perte de la clientèle qui est attirée par les avantages de l'automobile, le transport collectif souffre de faible rentabilité ${ }^{15}$ et de perte de vitesse commerciale due à la congestion du trafic, principalement en heures de pointe. En somme, la forte diffusion de l'automobile a eu de multiples effets négatifs sur l'environnement, la tranquillité des riverains, la congestion et le stationnement dans la ville dense :

- la congestion était confinée avant les années 2000 au niveau des principales artères de la ville et aux heures de pointe ; ces dernières années par contre, c'est la ville entière qui est plongée dans des embouteillages chroniques. La mauvaise gestion des carrefours qui sont dans leur quasi-totalité dépourvus des feux tricolores ${ }^{16}$ est pour une grande partie responsable de cette congestion et limite ainsi, la capacité des dessertes. Les travaux réalisés à partir de 2013 dans le cadre de l'aménagement urbain (trémies, aménagement de nouvelles voiries) n'ont fait que temporiser et déplacer les embouteillages. La volonté des autorités « d'adapter la ville à la voiture ${ }^{17}$ a donc échoué. Désormais, si cette politique avait donné ses résultats dans les pays développés pendant les années 1950 jusqu'aux années 1970, elle n'est plus opérante à l'heure actuelle. C'est plutôt une politique inverse qui s'insère dans le cadre du transport durable: elle consiste à « adapter la voiture à la ville » et doit être poursuivie. Autrement dit, il s'agit de développer des modes de transport propres, moins consommateurs d'espace et qui répondent aux attentes des usagers ;

- la raréfaction des espaces de stationnement est devenue un problème de plus en plus aigu. Il devient réellement

\footnotetext{
${ }^{15}$ Les entreprises de transport collectif font face à des besoins de financement colossaux et à une stagnation des recettes, ce qui réduit les possibilités d'amélioration des services. Dans les pays développés, les entreprises de transport ont recours aux fonds publics et à d'autres ressources telles que des contributions des activités économiques présentes sur l'agglomération desservie (par exemple le Versement Transport en France) pour équilibrer leur budget.

${ }^{16}$ Avant la mise en place du plan de circulation dont les travaux sont en cours, la ville ne dispose que d'un seul feu tricolore!

${ }^{17}$ Expression du président Français Pompidou (1911-1974).
} 


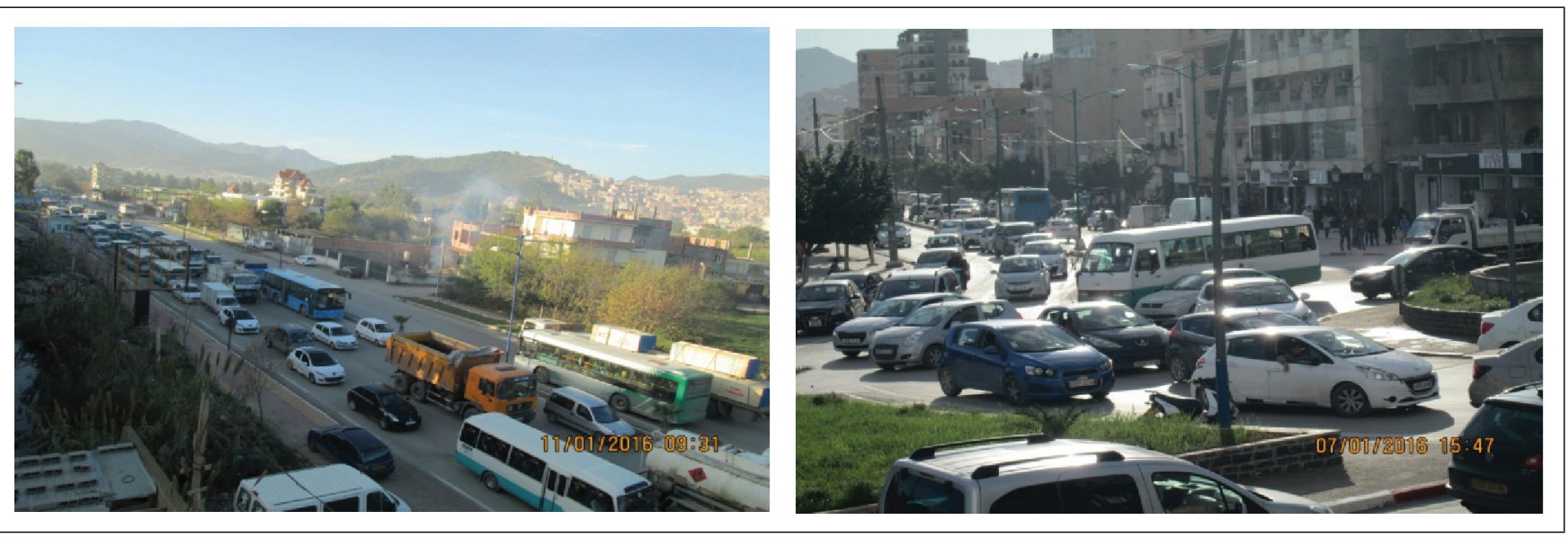

Fig 2. Photo d'embouteillages dans la ville de Bejaia

Source : photos prises par nos soins, 2016.

difficile de se garer faute d'espaces dédiés aux parkings. L'insuffisance des espaces de stationnement dans la ville de Bejaia n'est pas sans conséquence sur l'activité économique et sociale. L'enjeu du stationnement qui constitue la destination de tous les déplacements motorisés se résume dans l'expression no parking, no business. Les quelques espaces de stationnement disponible ${ }^{18}$ sont gérés par des jeunes sans-emploi qui procèdent à une sorte de « rackétage » des automobilistes en les obligeant à payer des sommes fixées à leur guise ;

- une nuisance et une pollution en nette augmentation dans le périmètre urbain qui altèrent la qualité de vie des citadins. Le bruit de la circulation est de moins en moins supportable puisque $80 \%$ de l'énergie acoustique est émise par les véhicules en circulation [14]. Les spécialistes fixent le niveau de bruit à 65 décibels $^{19}$ $[\mathrm{Db}(\mathrm{A})]$, au-delà de ce niveau le bruit est considéré comme très gênant, notamment pour le sommeil.

La pollution est le second problème épineux auquel sont confrontées les villes. À cet effet, la simple observation des faits et l'écoute des débats publics montrent que la réconciliation du transport avec l'environnement n'est pas pour demain [15]. Avec le processus de tertiarisation de l'économie et la délocalisation des activités industrielles vers les nouvelles zones industrielles (Taharacht et Elkseur), le transport demeure le principal pollueur.

En dépit de ces gênes qui sont multipliées par une forte utilisation de la voiture, l'opinion publique reste indifférente et peu encline à changer ses comportements vis-à-vis

\footnotetext{
${ }^{18}$ Ces espaces ne répondent pas aux normes requises et sont dans la majorité des cas exploités informellement et situés sur la chaussée réservée à la circulation!

${ }^{19}$ Le décibel A est l'unité la plus utilisée pour mesurer le bruit.
}

des modes de déplacement. De leur côté, les pouvoirs publics tardent à mettre en place un transport collectif de qualité susceptible de limiter les modes de déplacement dispendieux.

Cette situation des transports collectifs dans la ville n'est pas spécifique à cette dernière, mais c'est le cas de la majeure partie des villes algériennes. Les similitudes se résument dans la couverture spatiale limitée, la faible fréquence, la mauvaise régularité et surtout un accroissement rapide des opérateurs privés qui sont mal contrôlés. En bref, après la libéralisation du secteur vers la fin des années 80 , les problèmes se posent davantage en terme qualitatif [13].

Plusieurs caractéristiques du transport en commun dans la ville de Bejaia sont présentes dans plusieurs villes des pays en développement. En Asie du Sud-Est les villes fond face à des difficultés de mobilité dues à l'accélération d'une urbanisation mal maîtrisée, à l'augmentation du taux de motorisation conséquence de l'élévation du niveau de vie, et enfin à l'amplification des externalités négatives, notamment en termes de pollution et des accidents de circulation [16]. Dans les différentes villes d'Amérique latine les transports collectifs sont caractérisés par : la flexibilité d'exploitation, les formes de propriété atomisée ; le travail artisanal qui permet de réduire les coûts d'exploitation, le comportement corporatiste pour défendre les intérêts des transporteurs, une solidarité interne entre les exploitants [17]. Devant de telles difficultés et des coûts socio-économiques et environnementaux supportés par toute la communauté locale, le transport collectif sur site propre ${ }^{20}$ et les modes doux sont une réelle alternative pour accompagner la dynamique socio-économique que connaît cette ville. Afin d'y améliorer la mobilité, un plan de circulation est déterminé et

\footnotetext{
${ }^{20}$ Séparation du système de transport avec la circulation générale d'une façon partielle ou totale.
} 
un projet de tramway est envisagé. La nature et les objectifs de ces projets seront l'objet du développement suivant.

\subsection{Nouvelle offre de transport (tramway et plan de circulation)}

Par définition un transport de masse ou un mode lourd représente un mode de transport capacitaire et fiable et répondant aux objectifs de transport durable. Il existe quatre systèmes de transport de masse, qui sont : les modes ferrés (trains, tram-train, métro, tramway et LRT) et les modes routiers (BHNS à la française ou BRT voire Heavy BRT). Ce transport de masse est caractérisé par les éléments suivants : qualité de service, régularité, fiabilité, fréquences et densité suffisantes $[7,18,19]$. Dans ce type de transport collectif, le coût par passager est une fonction décroissante du nombre de passagers, ce qui se traduit par la présence des économies d'échelle.

Les rendements croissants liés à l'augmentation des utilisateurs dans le transport de masse s'expliquent par la réduction des coûts unitaires d'exploitation qui sont eux-mêmes dus à la diminution de la part des coûts fixes. À son tour, la forte densité et la croissance de la demande de déplacements sont favorables à ce mode de transport et facilitent l'amélioration des fréquences et de réduction des temps d'attente aux arrêts et des coûts du temps de déplacements.

Comme le montre la figure 3, le tramway de Bejaia est prévu sur la partie plate de la ville et sera réalisé en deux étapes. La première ligne indiquée sur la partie gauche de la carte est prévue à l'horizon 2020 et prendra en charge les flux est-ouest (centre-ville- Ihaddaden) et s'étalera sur une longueur de $7 \mathrm{~km}$ et comprend 14 stations. L'extension de la première ligne est envisagée à l'horizon 2030 et reliera le second pôle universitaire (Targa Ouzemour) au Carfour Naceria sur une longueur de 2,7 km et comprend 5 stations. En effet, les tracés choisis desserviront le secteur le plus dense de la ville (Ihaddaden) qui totalise 54580 habitants et les secteurs qui concentrent le plus d'emplois (rue de la liberté et zone industrielle) qui totalisent à eux seuls 36985 emplois [20]. Toutefois, le second secteur de concentration d'habitants de Sidi Ahmed avec 44465 habitants ne sera pas desservi par le tramway en raison des conditions géographiques de la zone caractérisée par le relief. Ce secteur sera relié à la ligne de tramway via la station polyclinique à l'aide du transport collectif traditionnel (bus de l'ÉTUB et privés et les taxis).

Le choix du tramway parmi d'autres modes de transport de masse pour l'amélioration de la mobilité dans les grandes villes algériennes ( à 100000 habitants) est motivé par l'atteinte des cinq objectifs suivants : desservir la demande de mobilité, maximiser l'intégration $\mathrm{du}$ transport en commun et améliorer sa qualité et son fonctionnement, minimiser les impacts environnementaux et maximiser la compatibilité avec le tissu urbain. Ces mégaprojets urbains décidés par les autorités centrales dans plusieurs villes algériennes sont confiés à l'EMA qui prend en charge leur exploitation. Le déploiement des transports publics (entreprises publiques de transport par autobus et par tramways) est en cours de réalisation à l'échelle nationale et touchera toutes les grandes villes. La capitale Alger bénéficie d'un programme spécial de développement des transports collectifs avec l'entrée en exploitation du métro en plus du tramway et du transport par autobus.

En parallèle au tramway, les bus privés et de l'ÉTUB ainsi que les Taxis desserviront les mêmes zones exploitées à l'heure actuelle. Autrement dit, en l'absence d'un plan de réorganisation du transport collectif traditionnel, celui-ci se

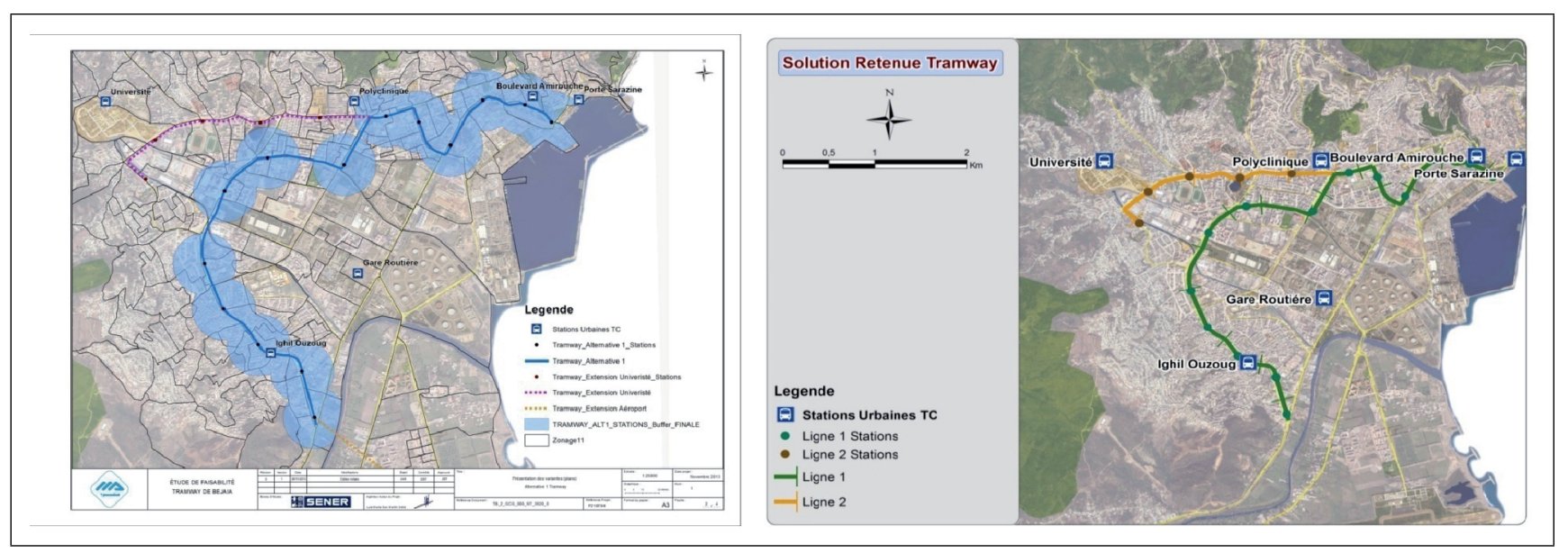

Fig 3. Carte du tracé du tramway de la ville de Bejaia

Source : Direction des transports de la wilaya de Bejaia, 2015 
place en position de concurrent au tramway. La connexion du tramway avec ces modes s'effectuera sur 4 pôles de connexions constitués par la structure actuelle du réseau de transport collectif et qui seront très limitrophes aux extrémités des deux lignes. Grâce à une fréquence de 4 minutes et à 19 stations qui seront distantes de 510 mètres en moyenne, les prévisions de transport à l'horizon 2030 fixent l'objectif de 8800 voyageurs en heure de pointe.

À travers l'exploitation d'une entreprise publique (ÉTUB) et la perspective de la réalisation du tramway, l'État vise à améliorer les déplacements urbains et à pousser les opérateurs privés, peu organisés et non respectueux des normes de service, à s'inscrire dans une démarche de qualité de service sur leur marché. Ce retour affirmé de l'État après plus de 20 ans d'absence quasi totale est jugé nécessaire et bon signe pour une meilleure gestion des déplacements en milieu urbain [12]. Cependant, la contractualisation qui définit les normes d'exploitation pour les opérateurs privés n'est pas clarifiée et ces derniers continuent d'exercer ce service public d'une façon très peu organisée.

La demande de transport est une autre composante essentielle dans la gestion de la mobilité qui doit être prise en compte dans le choix du type d'offre de transport. Pour contrôler la demande de transport dans la ville de Bejaia, un plan de circulation est arrêté. Ce dernier vise une utilisation optimale des espaces existants pour une meilleure gestion des flux de véhicule en accordant, notamment, au transport collectif et aux piétons une priorité dans cet espace urbain. Il prévoit particulièrement des aménagements des espaces de circulation et de stationnement pour automobile, des espaces pour piétons et une facilitation de la circulation des transports collectifs. Toutefois, ce plan de circulation n'envisage pas clairement la limitation des espaces dédiés à la circulation automobile au profit du transport collectif et des modes actifs (marche à pied et vélo), alors que les politiques de transport innovantes s'orientent vers la réduction de la place de l'automobile en ville. Ces politiques sont préconisées par la commission européenne sur les transports qui va même plus loin en envisageant le retrait progressif des véhicules qui utilisent des carburants traditionnels pour réduire la dépendance au pétrole, les émissions de gaz à effet de serre et la pollution sonore et atmosphérique [21].

En résumé, voici les principaux axes du plan de circulation prévu :

- un cadre réglementaire qui limite l'accès et le stationnement anarchique sur les principaux axes de la ville. Ce cadre vise, notamment, à réglementer l'accès des poids lourds et l'organisation des approvisionnements des centres commerciaux situés dans le centre urbain. Cette organisation va se concrétiser par des panneaux de

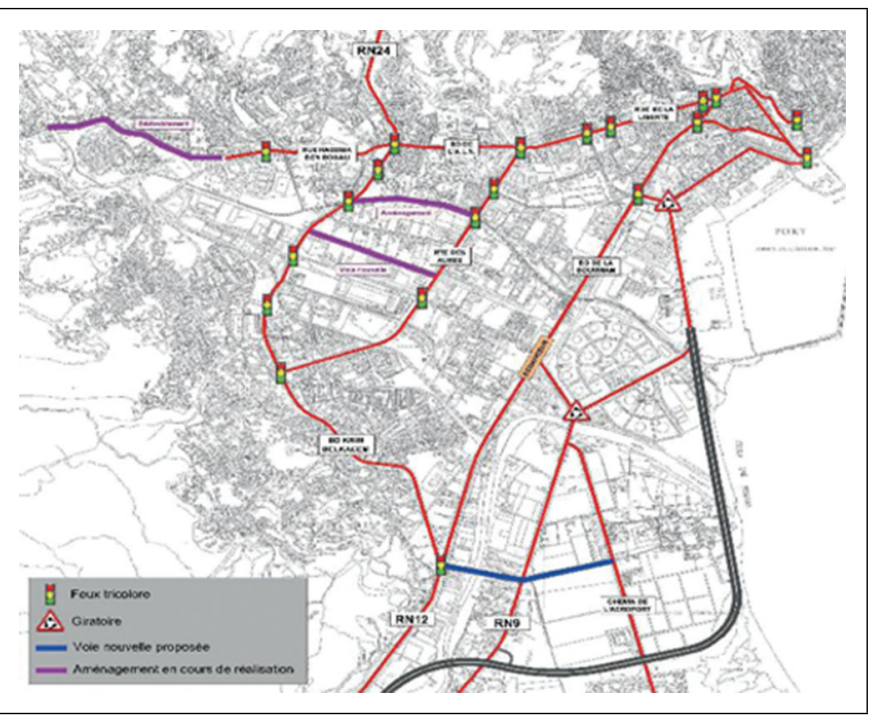

Fig 4. Carte d'aménagement de l'infrastructure - Synthèse des aménagements proposés

Source : direction des transports de la wilaya de Bejaia, 2015

signalisation et aussi par l'imposition de la force publique à travers l'intervention des agents de l'ordre ;

- des aménagements de carrefours les plus congestionnés qui seront dotés de signalisation tricolore et la réalisation de nouveaux accès routiers (Figure 4).

- la mise en place des parkings au centre-ville et l'installation des parcs relais sur les trois principales entrées de la ville qui sont destinés aux automobilistes en provenance des communes limitrophes. Ces parkings à proximité des transports collectifs qui seront utilisés moyennant un paiement visent à limiter les déplacements motorisés au centre-ville ;

- enfin, pour évacuer les automobilistes et les poids lourds en transit et éviter qu'ils passent par le milieu urbain, deux grands projets sont à ce titre envisagés. Le premier qui est à l'étude consiste à relier la RN 12 à la RN 24 et le second est en cours de réalisation et reliera le port de Bejaia à l'autoroute est-ouest (Figure 5). Certes ces projets sont très couteux pour le budget de l'État, mais ils constituent une bouffée d'oxygène pour cette ville asphyxiée par l'urbanisation mal contrôlée et par sa situation géographique caractérisée par la présence d'une chaîne montagneuse au sud-ouest et par la mer méditerranéenne au nord. Par ailleurs, la dynamique de son port de commerce situé au cœur même de la ville et classé au $2^{\text {eme }}$ rang des ports nationaux après celui de la capitale Alger provoque des flux importants de poids lourds qui acheminent des marchandises à partir et vers l'arrière-pays. Toutefois, l'accroissement des infrastructures routières que nous jugeons nécessaires peut provoquer un accroissement de la demande de 


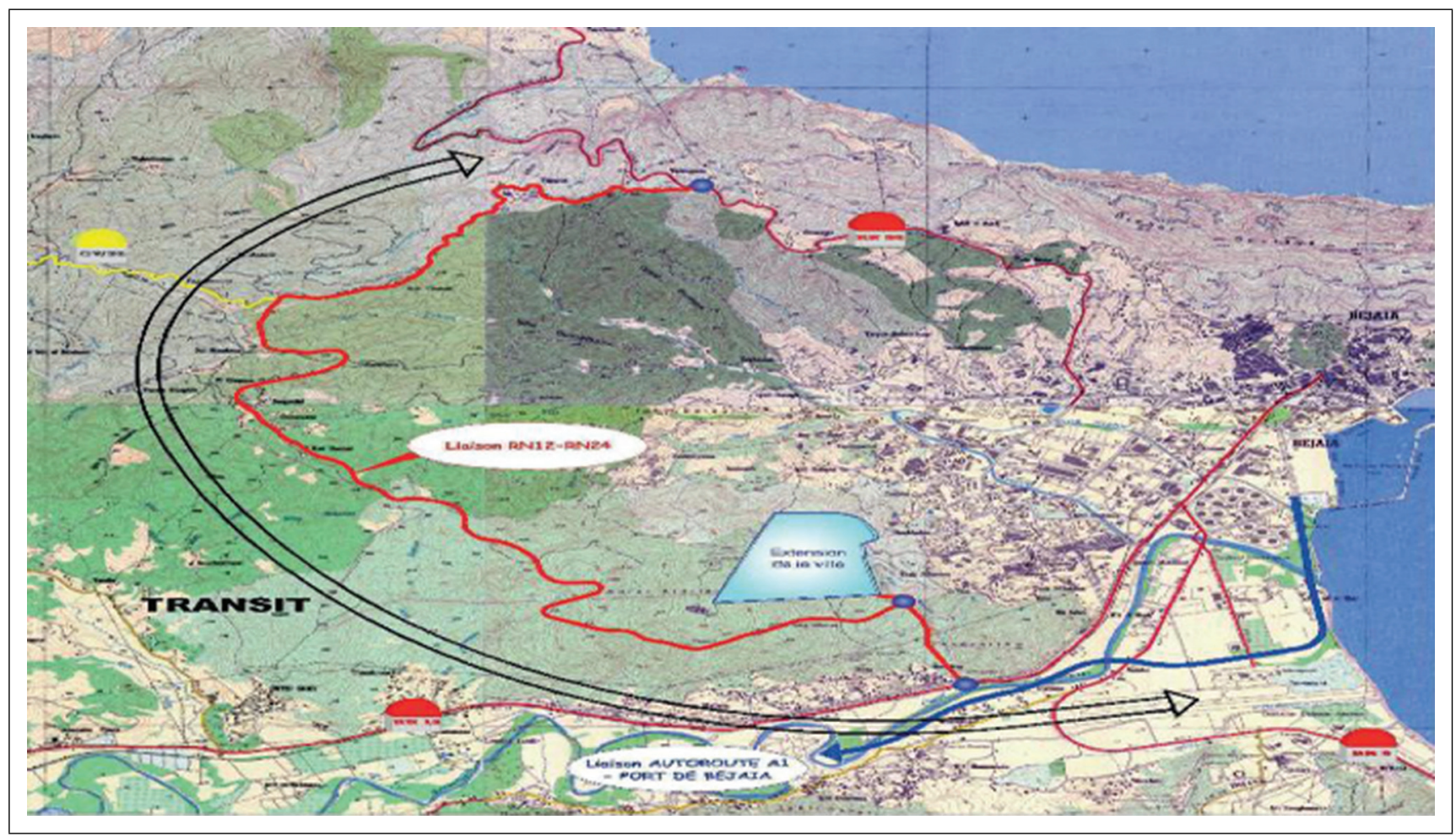

Fig 5. Carte de la hiérarchisation du réseau les 2 grands projets à l'étude

Source : direction des transports de la wilaya de Bejaia, 2015.

déplacement par automobile, ce qui est désigné par le paradoxe de Downs [22].

\section{La demande de transport : motifs et modes de déplacement}

Une meilleure connaissance de la structure de la demande est nécessaire avant d'envisager tout projet d'infrastructure de transport. Ainsi, l'objet de cette partie est d'identifier les modes, les motifs et les fréquences des déplacements en référence aux enquêtes réalisées dans la ville de Bejaia : Tous modes motorisés (TMM) et origine/destination $(\mathrm{O} / \mathrm{D})$ (2013) et Enquête ménage déplacement (EMD) (2006).

\subsection{Présentation des enquêtes $\mathrm{O} / \mathrm{D}$ et TMM.}

Les deux enquêtes $(\mathrm{O} / \mathrm{D}$ et $\mathrm{TMM})$ sont réalisées par le BETUR sur la mobilité urbaine et sur les motifs de déplacement dans la ville de Bejaia. Les enquêtes s'inscrivent dans le cadre du contrôle et de la gestion de la mobilité urbaine au sein de cette ville.

La première enquête sur les comptages des flux TMM vise à obtenir une image la plus exhaustive possible sur les déplacements motorisés durant la journée et pendant l'heure de pointe. Les différents véhicules recensés et les coefficients d'équivalences appliqués afin d'agréger les divers modes de transport sont présentés en tableau 2.

L'heure de pointe qui correspond à $9 \mathrm{~h} 45-10 \mathrm{~h} 45$ est ressortie de l'enquête journalière qui l'a précédée. Les comptages sont effectués sur les principaux axes routiers au sein de l'agglomération.

La date et la période des deux enquêtes correspondent aux jours ouvrables, entre $07 \mathrm{~h} 00$ et $19 \mathrm{~h} 00$ en dehors

Tableau 2 Les différents types de véhicules recensés lors de l'enquête TMM

Les Véhicules légers (VL) : Voitures particulières (VP) et Voitures utilitaires (VU)

Les Poids Lourds (PL) et Semi-remorques (SR)

Les Transports collectifs $(\mathrm{TC}):$ les autobus $(\mathrm{AB}=70$ à 100 places), les autocars ( $\mathrm{AC}=50$ places $)$, les minicars $(\mathrm{MC}=25$ à 35 places), les fourgons ( $\mathrm{F}=11$ à 22 places) et les Taxis ( $\mathrm{TX})$ Les 2 R (2 Roues)

Afin d'agréger les différents modes de transport pour n'exprimer qu'une mesure de débit de circulation, des coefficients d'équivalence ont été retenus lors de cette enquête : $\mathrm{VP}, \mathrm{Taxi}=1 \mathrm{U}$; $\mathrm{MC}, \mathrm{F}=1,5 \mathrm{U}$.; $\mathrm{PL}, \mathrm{VU}=2 \mathrm{U}$. ; $\mathrm{SR}, \mathrm{AC}, \mathrm{AB}=2,5$ U. ; $2 \mathrm{R}=0,3 \mathrm{U}$ 
des vacances, du congé annuel ou des autres événements pouvant perturber les déplacements de personnes.

La seconde enquête $\mathrm{O} / \mathrm{D}$ a comme objectif de déterminer la répartition du trafic entrant et sortant dans la ville de Bejaia par modes, par lieu de résidence et par motif de déplacement. La localisation des postes de l'enquête est au niveau des trois accès de la ville et cela pendant deux heures $(9 \mathrm{~h} 15-11 \mathrm{~h} 15)$ qui correspondent à la plage horaire de pointe déterminée lors de la première enquête TMM. En plus du comptage de l'ensemble des véhicules passant par le poste, les enquêteurs ont interviewé un échantillon de conducteurs sur leur lieu de résidence, à leur domicile, sur la fréquence de leur mobilité et les motifs de déplacements.

\subsection{Modes de déplacement}

L'enquête TMM est réalisée sur 23 postes de comptages, parmi lesquels six postes sont journaliers et 17 postes sont réservés uniquement pour l'Heure de pointe du matin (HPM)

Le débit journalier fourni par l'enquête est exprimé en équivalents voiture particulière (unités calculées selon les coefficients présentés en tableau 3 ) dans les deux sens et sur les six postes journaliers retenus. Cette agrégation est faite pour homogénéiser la mesure de débit de la circulation. Afin de calculer le débit de véhicules et le taux de remplissage pour les différents modes, nous avons transformé le débit par unités en tenant compte des coefficients d'équivalences utilisés par les enquêteurs.

À l'heure de pointe du matin, on remarque dans le tableau 3 que le cordon intérieur connaît le débit de véhicules le plus important (14 627) suivi du cordon périphérique (11 064) et enfin du cordon extérieur (4 951). Cette hiérarchie s'explique par le fait qu'à cette période de la journée (9h45-10h45), les populations des communes limitrophes et des quartiers périphériques de la ville ont fait déjà leur entrée au centre-ville ce qui a comme conséquence une forte demande de déplacement.

Tableau 3 Répartition modale du trafic par poste sur les deux sens à l'HPM (sur les trois cordons)

\begin{tabular}{lrrrrrr}
\hline & VP & \multicolumn{1}{c}{ TC } & VU & PL \& SR & 2 Roues & Total \\
Cor. int. & 10579 & 1477 & 1217 & 441 & 913 & 14627 \\
Cor. Pér. & 7510 & 1062 & 1114 & 948 & 430 & 11064 \\
Cor. ext. & 3098 & 762 & 484 & 504 & 103 & 4951 \\
Total & 21187 & 3301 & 2815 & 1893 & 1446 & 30642 \\
En \% & 69,14 & 10,76 & 9,19 & 6,17 & 4,72 & 100 \\
\hline
\end{tabular}

Source : Établi à partir des données fournies par le Bureau d'études des transports (BETUR).
Par ailleurs, le tableau montre que la voiture particulière représente plus de deux tiers du débit global sur les trois cordons $(70 \%)$, suivi des transports collectifs $(10,7 \%)$, $\mathrm{du}$ véhicule utilitaire $(9 \%)$, du poids lourd et des semi-remorques $(6 \%)$ et des deux roues $(4,7 \%)$.

Outre le débit de véhicules qui donne une idée globale sur les modes de transports utilisés et leur consistance, le taux de remplissage indique également le degré d'utilisation du transport collectif, mais aussi l'ampleur de la pollution engendrée par personne. Le tableau 4 montre que le taux de remplissage moyen est de 3,18 , avec une légère prépondérance du cordon extérieur. Cela s'explique par les taux de remplissage des minicars et des véhicules utilitaires qui sont très sollicités pour les déplacements à partir des quartiers périphéries et les communes limitrophes vers le centre-ville. Toutefois, le taux le plus élevé est enregistré par les minicars sur le cordon intérieur qui est dû à leur forte couverture de l'espace urbain et périphérique. Cependant, le faible taux de remplissage sur le cordon périphérique peut s'expliquer par l'excédent des places offertes au fur et à mesure qu'on se rapproche des extrémités des quartiers périphériques. Autrement dit, le taux de remplissage diminue progressivement en allant du centre-ville vers la périphérie en raison de la baisse de la demande.

Par ailleurs, le taux de remplissage des VP, VU croît légèrement quand on s'éloigne du centre (il s'améliore en longue distance); tendance qui n'est pas très stable pour les autres types de véhicules. Cette amélioration du taux de remplissage des VP et des VU sur longue distance peut s'expliquer, d'une part, par la faible couverture des zones éloignées du centre urbain par le transport collectif et de l'autre part, par une motivation économique et de moindres coûts rendue possible par les déplacements en groupe. Le taux de remplissage élevé pour les VU (plus de 3) est dû à l'utilisation mixte de ces véhicules pour les motifs professionnels, mais aussi pour les déplacements ordinaires en famille ou entre amis.

Enfin, le taxi reste le mode le moins sollicité sur les trois cordons probablement en raison de son coût élevé pour l'usager ${ }^{21}$ ce qui fait de lui un mode réservé à une certaine classe aisée de la population. Il est aussi utilisé occasionnellement pour les déplacements avec bagages vers les quartiers non desservis par les autres modes de transport collectif. Il est enfin utilisé pour son confort et pour des considérations sociologiques (sauvegarder l'intimité familiale et déplacement indépendant).

En somme, la prépondérance de la voiture particulière dans les débits constatés sur les trois cordons explique l'engouement à l'utilisation de l'automobile dans les déplacements urbains à Bejaia qui reflète dans une large

\footnotetext{
${ }^{21}$ Le coût moyen de déplacement est de 135 DA pour le taxi contre 15 DA pour le bus, selon l'EMD de 2006.
} 


\begin{tabular}{|c|c|c|c|c|c|c|c|c|c|c|c|}
\hline & VP & $\mathbf{V U}$ & Taxi & PL & SR & À Bus & À Car & M Car & Fourgon & 2 Roues & Total \\
\hline Cor.int. & 1,46 & 3,04 & 1,30 & 1,42 & 1,20 & 29,46 & 4,20 & 63,92 & 9,07 & 1,13 & 3,13 \\
\hline Cor. pér. & 1,51 & 3,04 & 1,56 & 1,34 & I & 54,98 & 9,74 & 21,16 & 4,08 & 1,11 & 2,99 \\
\hline Cor. Ext. & 1,59 & 3,48 & 1,48 & 1,67 & 2,42 & 12,57 & 2,66 & 52,83 & 5,17 & 1,20 & 3,76 \\
\hline Total & 1,50 & 3,11 & 1,43 & 1,45 & 1,32 & 24,01 & 4,87 & 39,58 & 6,31 & 1,13 & 3,18 \\
\hline
\end{tabular}

mesure la situation de la majeure partie des grandes villes algériennes. Cet engouement est dû aux facteurs d'ordres économiques, sociaux et culturels (amélioration du pouvoir d'achat, faiblesse du prix de carburant, découverte du confort, prestige...), mais surtout à la qualité insuffisante du transport collectif.

\subsection{Motifs de déplacement}

Dans la deuxième enquête $\mathrm{O} / \mathrm{D}$ réalisée pendant la période de 9 h 15 - 11 h 15, un échantillon de conducteurs des véhicules particuliers (VP) est interviewé sur les motifs et la fréquence de leurs déplacements. Sur un flux total de 6715 voitures particulières qui ont fait leur entrée pendant la période de $9 \mathrm{~h} 15-11 \mathrm{~h} 15$ sur les trois accès que contient la ville, 902 conducteurs sont interviewés.

La figure 6 montre que le principal motif de déplacement des conducteurs interviewés est le domicile-travail avec $37 \%$, suivi des déplacements secondaires et non obligés $(18 \%)$, des déplacements travail-travail $(12 \%)$ et du travail-domicile (10\%) qui est beaucoup moins que domicile-travail, puisqu'on est à la période du matin. Les déplacements les moins représentés sont liés au domiciledémarches administratives et domicile-études (5\%).

Les déplacements obligés (travail-domicile, domicileétudes ...) sont les plus importants et représentent 66,5\% des déplacements enquêtés. Les déplacements réguliers (ou obligés) sont plus adaptés au développement d'un transport collectif de masse, puisque la demande est importante et plus stable.

Les déplacements hétérogènes ou non obligés (Domicile-Santé, Domicile-Démarches administratives...) représentent 33,4\% du total de l'échantillon. Ces résultats confirment dans une large mesure les motifs de déplacement constatés lors de l'EMD de 2006 avec $76 \%$ pour des motifs obligés et $24 \%$ pour les motifs non obligés ${ }^{22}$.

\footnotetext{
${ }^{22}$ Toutefois, il y a lieu de préciser que l'EMD est réalisée auprès des ménages de la ville de Bejaia, alors que l'enquête O/D concerne uniquement les conducteurs des VP. Pour cette raison, les résultats ne sont pas parfaitement comparables.
}

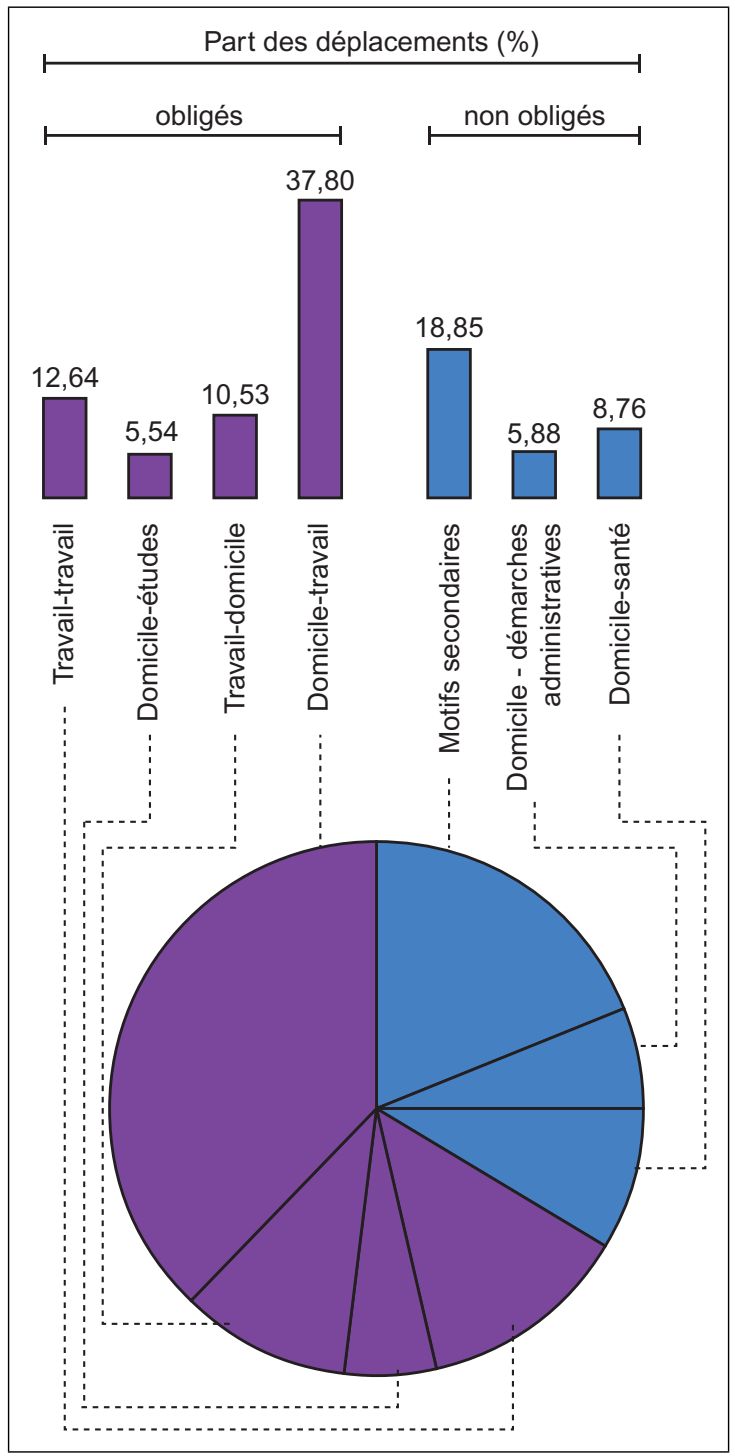

Fig 6. Graphe des motifs de déplacement Source : Établi à partir des données fournies par le BETUR, 2013.

En termes de fréquence des déplacements des conducteurs, la mobilité quotidienne (trois fois par semaine et plus) est la plus importante avec un taux de 70,5\% (Figure 7). Les déplacements occasionnels (une à quatre fois par mois) 
viennent en deuxième position avec un taux de $27.2 \%$. Enfin, les déplacements rares (moins d'une fois par mois) sont de l'ordre de $2,2 \%$. Cette hiérarchie est toute à fait logique puisque la fréquence de déplacements est liée aux motifs qui sont dans notre cas dominés par des déplacements homogènes et obligés.

La troisième composante de l'enquête $\mathrm{O} / \mathrm{D}$ est liée au lieu de résidence des utilisateurs de la voiture particulière.

La figure 8 montre que $53,1 \%$ de l'échantillon enquêté correspond à une population qui réside dans les communes limitrophes. Les résidents du périmètre urbain représentent $34,7 \%$, tandis que les résidents hors wilaya viennent en dernière position avec $12,2 \%$.

Les utilisateurs de la voiture particulière en provenance des régions hors périmètre urbain (autres communes et hors wilaya) ne seront pas desservis par le projet de tramway qui couvre uniquement le centre urbain qui est à l'origine

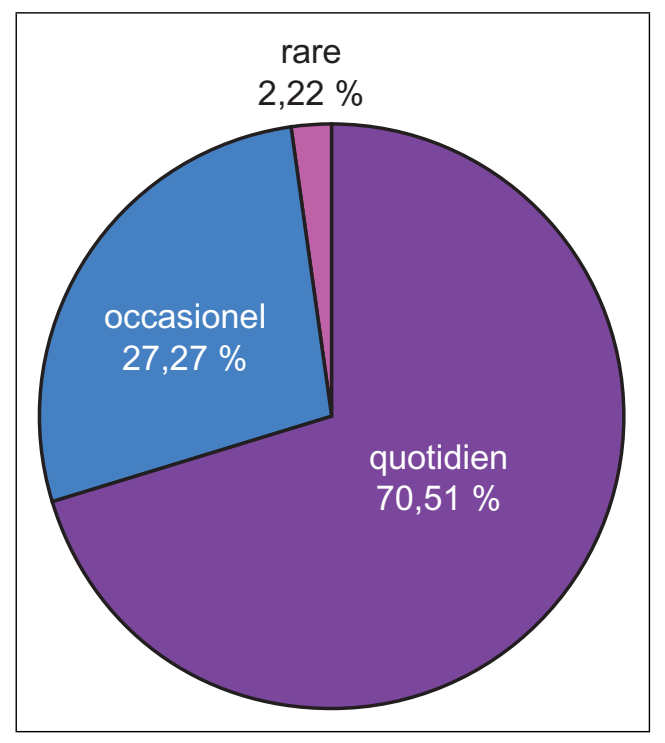

Fig 7. Graphe de la fréquence des déplacements (part en pourcentage)

Source : établi à partir des données fournies par le BETUR, 2013.

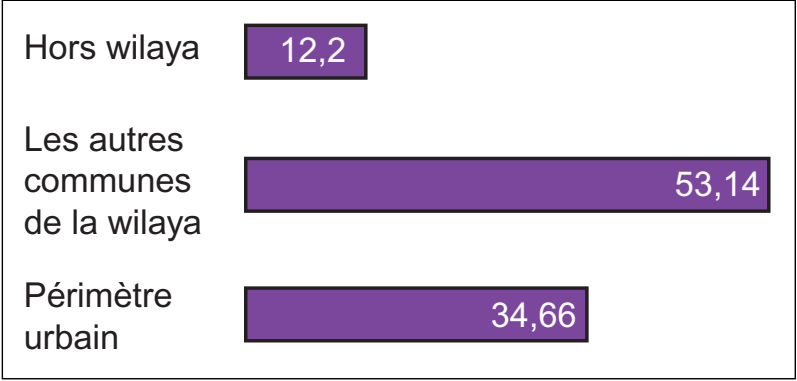

Fig 8. Graphe du lieu de résidence des usagers de la voiture particulière (en pourcentage)

Source : établi à partir des données fournies par le BETUR, 2013. de $34 \%$ des flux sur les trois accès que contient la ville. Les communes limitrophes continueront d'être desservies par les transports collectifs traditionnels, mais aussi par voie ferroviaire ${ }^{23}$ pour celles qui sont sur la vallée de la Soummam.

\section{L'offre de transport collectif et les attentes de la population}

La fréquentation du transport collectif dépend de la quantité (nombre de places offertes) et de la qualité de service. Cette dernière est le résultat d'une organisation globale du système de transport qui doit être orienté dans une logique de développement durable. Nous nous interrogeons dans la présente partie sur les apports du tramway en termes de satisfaction de la demande et de la performance environnementale.

L'amélioration des conditions de mobilité urbaine dans la ville de Bejaia nécessite la mise en place d'un système de transport qui tient compte des motifs, des modes et de l'intensité des déplacements. La structure actuelle de l'offre de transport collectif est incapable de répondre à une demande sans cesse croissante constituée par la mobilité quotidienne des résidents comme des non-résidents. L'idée d'une nouvelle offre de transport capacitaire est inéluctable, notamment vers et à partir des quartiers périphériques fortement urbanisés. Le projet de tramway s'inscrit dans cette optique, car il constitue un mode de transport de masse capable de s'adapter à des flux très importants en augmentant la cadence des rotations ou en mettant en place davantage de rames. Pour cette raison nous considérons que le tramway constituera une alternative innovante capable d'améliorer les déplacements sur les axes qui seront desservis par ce nouveau mode de transport. Le réseau de transport collectif formera une arête de poisson dans laquelle le tramway constitue la structure principale à côté des autres modes traditionnels qui en attendant leur réorganisation seront en position de concurrents. Autrement dit, en l'absence d'un projet de réorganisation du réseau de transport actuel, l'axe traversé par le tramway sera desservi aussi par les modes traditionnels. Les minibus joueront un rôle capital dans ce réseau en desservant les zones périphériques difficiles d'accès en raison des routes exiguës et inadéquates pour les bus de grandes capacités.

Prévu sur une longueur de 9,7 km à l'horizon 2030, le tramway desservira trois zones les plus denses sur les deux cordons intérieur et périphérique. Il couvre notamment les deux orientations des flux les plus importants : Est/Ouest

\footnotetext{
${ }^{23}$ Cette voie ferroviaire qui longe une partie des communes situées sur la vallée de la Soummam sera renforcée par un projet de dédoublement dont les travaux sont en cours de réalisation.
} 
Tableau 5 Prévisions de la demande de transport par modes en heure de pointe.

\begin{tabular}{lll}
\hline Modes de transport & Horizon 2020 & Horizon 2030 \\
\hline Bus privés & 21334 & 30141 \\
Bus ÉTUB & 1634 & 2660 \\
Tramway & 5049 & 8821 \\
Total & 28017 & 41622 \\
\hline
\end{tabular}

Source : EMA, étude de faisabilité d'une ligne de tramway de la ville de Bejaia, février 2014.

et Sud-Ouest/Nord-Est. Toutefois, pour des considérations techniques et géographiques la seconde zone la plus dense au sein de la ville (Sidi Ahmed) ne sera pas desservie par le tramway et devra être connectée à celui-ci par des bus privés et de l'ÉTUB au sein de la station Polyclinique.

D'après la structure actuelle du réseau de transport urbain, les opérateurs privés couvrent tous les quartiers périphériques de la ville à côté de l'ÉTUB qui se limite à quelques lignes urbaines. Ces opérateurs partagent donc la demande de transport qui selon les prévisions faites dans le cadre de l'étude de faisabilité du projet de tramway sera répartie comme suit (aux horizons 2020 et 2030) :

Étant donné que les deux lignes convergent vers le centre-ville, le tramway aura des effets positifs sur cette zone qui est la plus marquée par des problèmes de mobilité (déplacements difficiles des automobilistes et insuffisance des espaces de stationnement sur les principaux axes : Liberté, ALN, Hassiba Ben Bouali, Krim Belkacem). Le projet est donc susceptible de soulager ces axes les plus fréquentés au centre urbain ${ }^{24}$. L'atout majeur du tramway réside dans son évolution sur un espace réservé, ce qui contribue à la réduction du temps de déplacement qui est un critère important dans la mobilité urbaine.

Toutefois, les divers objectifs assignés à ce projet (satisfaction de la demande, réduction des impacts environnementaux, intégration avec le réseau de transport en commun, requalification des lieux traversés) dépendent de l'implication du citoyen et de la mise en place du plan de circulation qui vise à maîtriser et à orienter la structure des déplacements. Contrairement à cela, le projet n'a pas été présenté à la population qui n'est pas informée ni sur les motivations du choix de ce mode ni sur son tracé et stations envisagés ni même sur les objectifs qui lui sont assignés. De son côté, le plan de circulation pris

\footnotetext{
${ }^{24}$ Les deux enquêtes $\mathrm{O} / \mathrm{D}$ et TMM montrent que $98 \%$ des déplacements urbains et interurbains sont orientés vers l'intérieur de la ville et $59 \%$ des déplacements inter wilaya (en transite) sont aussi attirés par une destination à l'intérieur de la ville.
}

en charge par la commune de Bejaia tarde à voir le jour. Mise à part la réalisation des feux tricolores au niveau des principaux carrefours dont les travaux restent en cours, les autres variantes du plan de circulation ne sont pas entamées.

À cet égard, l'étude sur la participation citoyenne aux projets de tramways en Algérie réalisée par Alili $\mathrm{A}$, et Belkaid E, (2013) montre que ces derniers rencontrent des obstacles essentiellement d'ordre social [9]. La réalisation du tramway d'Alger a été bloquée à plusieurs reprises par les habitants qui ont contesté l'installation des stations à proximité de leur cité. À Oran, deuxième ville du pays, le tramway a connu d'autres types de problèmes qui sont dus à l'installation des billetteries plus ou moins loin des stations et à la contestation des commerçants qui se plaignent d'une baisse d'activité engendrée par la difficulté d'accès de leurs clients en véhicules. À Constantine, le prix du ticket fixé à 40 DA pour seulement $9 \mathrm{~km}$ est jugé hors de portée pour le citoyen, comparativement au prix pratiqué à Alger qui est de $50 \mathrm{DA}$ sur un trajet de $16 \mathrm{~km}$. Enfin, le problème de sécurité des piétons et des véhicules qui traversent les tracés des tramways reste le point commun à ces projets lors du passage des trains.

La seconde étude réalisée par Labaoui $\mathrm{Y}$, et Abass L, (2014) sur l'impact du projet du tramway d'Alger montre aussi plusieurs limites de ce projet [10]. L'étude souligne particulièrement que la congestion urbaine persiste après l'exploitation du tramway. Cette congestion est accentuée par la réduction des espaces de circulation et de stationnement des voitures suite à la réalisation du projet. En plus, le projet de tramway a pénalisé les commerçants qui ont vu leur activité baissée en raison des difficultés d'accès pour les piétons et pour le manque d'espace de stationnement. Cependant, les populations enquêtées déclarent que le cadre et les conditions de vie se sont améliorés avec une réduction du bruit, de la pollution et une nouvelle image plus esthétique des quartiers donnée par les aménagements effectués.

Ces évaluations sur les projets de tramway déjà réalisés en Algérie même si elles ne sont pas exhaustives, montrent que les objectifs assignés à ces projets ne sont pas optimisés. Les insuffisances ainsi constatées interpellent les autorités pour prendre les mesures nécessaires pour que le tramway de Bejaia soit une infrastructure structurante qui conciliera entre l'attractivité et les problèmes de mobilité dans cette ville.

Toutefois, le point faible du projet de tramway de Bejaia reste sa desserte d'un espace très limité. D'un côté, le second pôle de concentration de la population qui est Sidi Ahmed ne sera pas desservi en raison des conditions géographiques du secteur qui ne s'apprête pas à accueillir ce genre de mode de transport. De l'autre côté, le projet n'aura pas un impact direct sur la limitation des flux de voitures particulières 
en provenance des communes limitrophes qui demeureront desservies par le mode transport traditionnel.

\section{Conclusion}

Nos propos dans le présent article sont limités au système de transport ou plus particulièrement à la capacité du transport de masse (tramway) et du projet de plan de circulation à améliorer la mobilité dans la ville de Bejaia. La question de l'offre et de la demande de transport étudiée dans le présent travail est d'actualité dans les pays en développement qui connaissent une mutation démographique et urbanistique accélérée. Toutefois, les études récentes relatives au système de transport traitent davantage des domaines tels que la tarification, le calcul économique, les effets environnementaux, les coûts sociaux, la gestion et le financement des systèmes de transport. Le contexte de l'étude ainsi que l'actualité des investissements envisagés par les autorités justifient le choix de notre approche.

Nos apports relatifs à l'objet de l'étude montrent que l'offre du transport traditionnel est en déphasage par rapport à la demande de mobilité quotidienne qui ne cesse d'augmenter. Par ailleurs, il y a une sorte de dualisme du système de transport qui voit le développement des nouveaux modes (bus et tramway) à côté des transporteurs traditionnels privés (minibus, fourgon, taxi). Le retour de l'État dans le cadre des nouveaux modes à travers les projets de transport de masse et la réhabilitation du transport collectif par bus est motivé par sa volonté de pousser les opérateurs privés à améliorer la qualité de leur prestation sur leur propre marché. Toutefois, cette stratégie reste un vœu pieux et est loin d'être réalisable, car plusieurs aspects du système doivent être repensés pour réussir le transfert modal (réduire l'utilisation de la voiture particulière au profit $\mathrm{du}$ transport en commun) et encourager les modes actifs (vélo et marche à pied). Autrement dit, il est nécessaire d'adopter une solution intégrée qui tient compte des divers aspects qui relèvent de la mobilité en agissant à la fois sur la demande et sur l'offre de transport. En somme, les projets en cours tels qu'ils sont prévus ne seront pas en mesure de concilier la fonction de déplacement et l'attractivité de la ville de Bejaia. En effet, ces projets ne sont pas conçus avec la participation du citoyen qui n'a pas la moindre information sur les aspects techniques et opérationnels ce qui les expose au rejet de celui-ci dès le début des travaux de réalisation. Par ailleurs, les impacts socioéconomiques et environnementaux de ces projets dépendent dans une large mesure des aménagements à effectuer le long du tracé et de ceux prévus dans le plan de circulation. Ces aménagements qui sont intégrés dans le cadre du Plan de Développement et d'Aménagement Urbain communal et intercommunal semblent toutefois négligés certains éléments clés qui sont indispensables pour une mobilité durable dans cette ville. Nous tenons à cet égard à préciser ces éléments clés qui ne sont pas pris en compte dans la nouvelle offre de transport et dans le plan de circulation et qui sont les suivants:

- le passage d'un régime d'autorisation à un régime de contractualisation qui définit les droits et les obligations des prestataires de transport privés, ou ce que nous avons appelé les transporteurs traditionnels. Certes une telle réforme sera combattue par le refus et la contestation des opérateurs privés qui dominent le marché à l'heure actuelle, mais c'est une réforme nécessaire si l'on veut améliorer la qualité de service. Contrairement à cela, les pouvoirs publics veulent changer l'image du transport collectif sans le concours des transporteurs privés qui sont totalement oubliés dans le système de transport envisagé;

- l'inter-modalité qui est indispensable pour optimiser le système de transport est aussi le grand élément oublié dans la structure du réseau envisagée. Cette intermodalité est seule capable d'augmenter la fréquentation des transports collectifs. Elle est assise notamment sur la réorganisation des réseaux de transport et l'intégration tarifaire. La réorganisation des réseaux consiste à assurer une meilleure couverture géographique et une interconnexion entre les modes traditionnels qui assurent le transport sur les quartiers limitrophes et le transport capacitaire ou le tramway prévu au centre-ville. Quant à l'intégration tarifaire, elle permet aux usagers de voyager à l'aide d'un seul titre de transport sur les différents modes et vers les différentes destinations. Cependant, dans le contexte actuel caractérisé par l'existence d'une multiplicité d'opérateurs privés il est très difficile d'asseoir une tarification unique. Toutefois, elle peut être réalisée graduellement en l'appliquant sur chaque ligne de transport pour être généralisée à terme sur tout le réseau urbain ;

- la réduction des espaces dédiés à la circulation automobile et des lieux de stationnement au bénéfice des transports collectifs et des modes doux et pour d'autres usages tels que les espaces verts. Un meilleur partage modal des espaces oblige les automobilistes à solliciter ces modes vertueux. Certes le plan de circulation a pris en charge certains points relatifs à la gestion du stationnement, à la création des parcs relais, à la création de nouveaux passages routiers, à des déviations autoroutières, à l'amélioration de la signalisation dans les carrefours, mais il ne prévoit pas une stratégie qui vise le partage modal des espaces au sein de l'agglomération ;

- enfin, compte tenu de l'importance des véhicules utilitaires en circulation dans la ville de Bejaia, il est nécessaire de passer à une meilleure distribution des marchandises. La rationalisation de la distribution des 
marchandises consiste à mieux remplir les véhicules et à réduire le nombre de tournées en mettant en place des centres de distribution ou des plates-formes logistiques à l'extérieure de la ville.

En somme, les concepteurs des projets de nouvelle offre de transport urbain sont interpellés pour tenir compte des expériences des autres villes algériennes qui ont connu la mise en exploitation des infrastructures de tramway, mais aussi des spécificités de la ville de Bejaia en matière de structure des déplacements et de sa situation géographique. À cet égard, notre analyse nous a permis de conclure que le projet de tramway de Bejaia ne règle que partiellement les problèmes de mobilité en raison de la non-desserte $\mathrm{du}$ second secteur de concentration des habitations (Sidi Ahmed), mais aussi des communes limitrophes qui sont à l'origine des flux importants de voitures particulières. De plus, l'étalement urbain mal contrôlé engendre de nouveaux besoins de déplacement qui sont souvent mal pris en charge par les transports collectifs ce qui favorise l'utilisation de la voiture individuelle. Toutefois, la problématique de l'étalement urbain et le rôle, notamment, des politiques d'aménagement du territoire qui ne sont pas traités dans la présente étude feront l'objet de nos prochains travaux. En effet, vu l'importance des politiques urbaines dans la structuration des réseaux de transport, nous essayerons d'analyser la place qu'occupe la fonction de mobilité dans les politiques d'aménagement du territoire en Algérie à travers, notamment les plans de développement nationaux, régionaux et locaux.

Remerciements Le présent article est réalisé à l'occasion de mon stage de 15 jours effectué au mois de mai 2015 à l'Ifsttar (Paris).

Je tiens à remercier tous les chercheurs et les doctorants de cet institut pour leurs remarques et orientations lors de ma présentation au séminaire de DEST. Je remercie très particulièrement $\mathrm{M}$. Jean-Loup Madre, maître de recherche à l'institut pour ses pertinentes contributions à la finalisation de cet article.

\section{Bibliographie}

1. Kassi-Djodjo, I (2010) Rôle des transports populaires dans le processus d'urbanisation à Abidjan. Les Cahiers d'Outre-Mer 25 : 391-402.

2. Roger, C (2007) Dynamique de la motorisation et usage de l'automobile en France : l'Île-de-France en perspective. Thèse de Doctorat en Sciences Économiques, Université de Paris I-Panthéon-Sorbonne.

3. Crozet, Y, Joly, I (2003) De l'hypothèse de la constance du budget temps de transport à sa remise en cause : une double interpellation des politiques de transport. Rapport de la recherche pour le PUCA.

4. Godard, X (2008) Obstacles à la mobilité durable dans les villes méditerranéennes, convergences et variété des contextes et trajectoires. Communication à la conférence Codatu XIII, Ho Chi Minh, novembre 2008.

5. Khelladi, M (2003) Analyse du système de transport urbain dans une ville moyenne : cas de Bejaia. Projet de recherche, Université de Bejaia.

6. Godard, X (2008) Les déplacements urbains en méditerranée. Travaux issus du séminaire régional sur les déplacements urbains en méditerranée, Skhirat, Maroc, 22 et 23 janvier 2008.

7. Mirabel, F, Reymond, M (2013) Économie des transports urbains. La découverte, Paris, $125 \mathrm{p}$.

8. Mahieux, A (2014) Economic assessment of strategic transport policies in a context of sustainable development: which innovative solutions? Application to the Syndicat Mixte des Transports Artois-Gohelle area. Thèse de Doctorat, Université de Lille I.

9. Alili, A, Belkaid, E (2013) Le rôle de la participation citoyenne dans l'adhésion aux projets urbains : cas des tramways en Algérie. Communication au colloque international, Université d'Annaba (Algérie).

10. Labaoui, Y, Abass, L (2014) Effets de l'introduction de nouveaux modes de transport sur la mobilité urbaine entre le centre et la périphérie d'Alger : impact du projet de tramway. Communication au colloque international, Université de Batna (Algérie).

11. Wiel, M (1999) La transition urbaine, ou le passage de la ville pédestre à la ville motorisée. Mardaga, Bruxelles, $149 \mathrm{p}$.

12. Boubakour, F (2008) Les transports urbains en Algérie face aux défis du développement durable: sur les problèmes rencontrés et les solutions proposées. Communication à la conférence de CODATU XIII, Ho Chi Minh, novembre 2008.

13. Boubakour, F, Merazga, A (2006) Planification du transport urbain et développement durable: Quelques réflexions à propos de la ville de Batna (Algérie). Transports 437 : 175-181

14. Marcadon, J, Auphan, E, Chesnais, M (1997) Prépas géographiques : les transports. Armand Colin, Paris, $215 \mathrm{p}$.

15. Meyronneinc, J-P (1998) Le transport face à l'environnement. Éditions CELSE, Paris, 179 p.

16. Nguyen, $\mathrm{T}$ (2005) Un modèle vietnamien de transport urbain : utopie ou réalités? Thèse de Doctorat en Sciences Economiques, Université Lumière Lyon II.

17. Rosa, V (1993) La réorganisation du système de transport collectif de surface à Caracas à partir de la mise en service du métro. Thèse de Doctorat Multidisciplinaire du Nouveau Régime, Université de Paris XII- Val-de-Marne.

18. CERTU (2013) Choisir un mode de transport capacitaire, l'exemple de villes méditerranéennes. Collection dossiers $\mathrm{N}^{\circ} 266$.

19. Bailly, J-P, Stathopoulos, N (2000) Les enjeux du transport public dans les villes européennes. Éditions presses ponts et chaussées, Paris, $397 \mathrm{p}$.

20. BETUR (2013) Filiale de l'Entreprise Métro d'Alger (EMA). Diverses statistiques.

21. Commission européenne (2011) Feuille de route pour un espace européen unique des transports : vers un système de transport compétitif et économe en ressources. Livre blanc, Bruxelles.

22. Litman, T (2011) Generated traffic and induced travel. Papier de recherche, Victoria transport policy institute. 